\title{
Efficient Light Harvesting of a Luminescent Solar Concentrator using Excitation Energy Transfer from an Aggregation-Induced Emitter
}

\author{
James L. Banal, Kenneth P. Ghiggino, and Wallace W. H. Wong \\ Received (in $X X X, X X X)$ Xth $X X X X X X X X X 20 X X$, Accepted Xth $X X X X X X X X X 20 X X$ \\ ${ }_{5}$ DOI: 10.1039/b000000x
}

The compromise between light absorption and reabsorption losses limits the potential light conversion efficiency of luminescent solar concentrators (LSCs). Current approaches do not fully address both issues. By using the excitation 10 energy transfer (EET) strategy with a donor chromophore that exhibits aggregation-induced emission (AIE) behaviour, it is shown that both transmission and reabsorption losses can be minimized in a LSC device achieving high light collection and concentration efficiencies. The light harvesting 15 performance of the LSC developed has been characterized using fluorescence quantum yield measurements and Monte Carlo ray tracing simulations. Comparative incident photon conversion efficiency and short-circuit current data based on the LSC coupled to a silicon solar cell provide additional ${ }_{20}$ evidence for improved performance.

Geometrical solar concentrators used in photovoltaics (PV) typically use an intricate system of mirror/lenses and tracking systems to focus sunlight on a high-performance solar cell which can reduce the amount of PV material required and lower the cost 25 of PV installations. However, the added expense of an electronic tracking system contributing to installation costs to maintain high concentration, bulky configuration, and solar cell heating is unattractive toward building-integrated photovoltaics (BIPV). Luminescent solar concentrators (LSCs) are low-cost alternative 30 concentrating systems that have been proposed thirty years ago. ${ }^{1}$ A typical LSC consists of a transparent waveguide that contains a low concentration of organic fluorophores, which are commonly laser dyes. These dyes absorb light and produce emission typically with high fluorescence quantum yield. Ideally, a large 35 fraction of these emitted photons are trapped within the waveguide and concentrated to the substrate edges by total internal reflection. This concentrated light can then be used for energy conversion in photovoltaic cells.

The performance of LSCs that use laser dyes has been limited by 40 reabsorption losses due to the small Stokes shifts that are inherent to dyes with rigid molecular structures. An obvious solution to this problem is to use low concentrations of dyes in hosts. However, this means less light can be absorbed by the LSC at a given device thickness increasing transmission loss. Increasing 45 the thickness of the LSC can reduce the transmission loss but at the cost of increased reabsorption stemming from tail absorption. ${ }^{2}$
Another approach to reduce reabsorption is to use excitation energy transfer (EET) to induce a large Stokes shift. The EET approach can be achieved in covalent (macromolecule energy 50 gradient) or non-covalent (dye cocktails) molecular systems. Covalent EET approaches have involved dendrimers that are substituted with a number of chromophores to create an intramolecular energy gradient. Several dendrimers have been reported to achieve a large Stokes shift to reduce reabsorption. ${ }^{3-5}$

${ }_{55}$ However, the fluorescence quantum yield of these dendritic materials are relatively low and synthetic accessibility of candidate dendritic materials can also limit their application for LSCs.

The non-covalent EET approach uses two or more dyes which 60 absorb light over a wide range of wavelengths but most of the emission of the LSC, if not all, originates from the dye that emits at the longest wavelength. ${ }^{6,7}$ The concentration of the EET donor and acceptor species in the host matrix is kept low to avoid aggregation effects and reabsorption but must be sufficient for 65 energy transfer to occur between the dyes. Low concentrations limit the absorption efficiency of the LSC and a thicker LSC is then required to maximize absorption but at the cost of increased reabsorption. ${ }^{6}$ Moreover, if there is significant overlap of the absorption spectra of the donor and acceptor, reabsorption losses 70 from both species can result. Having well separated absorption spectra of donor and acceptor in the LSC should improve the light harvesting of the LSC while keeping reabsorption to a minimum. ${ }^{8}$ Optimizing parameters affecting efficient energy transfer: chromophore concentration in film, energy transfer 75 efficiency, and quantum yield, is still a challenge. ${ }^{6,9}$ In this work, we have made improvements to the EET strategy by using, in high concentration in the LSC to harvest light efficiently, a donor chromophore which is insensitive to concentration quenching and shows a large Stokes shift. ${ }^{10}$ This donor material, 2-(480 (diphenylamino)phenyl)-3,3-diphenylacrylonitrile (DPATPAN), exhibits aggregation-induced emission behaviour and is used in conjunction with the high quantum yield acceptor dye 4(dicyanomethylene)-2-tert-butyl-6-(1,1,7,7-tetramethyljulolidyl9-enyl)-4H-pyran (DCJTB) at low concentration. The chemical 85 structures of the chromophores are shown in Figure 1a. This work demonstrates an EET strategy that minimizes reabsorption and transmission losses simultaneously. 
(a)

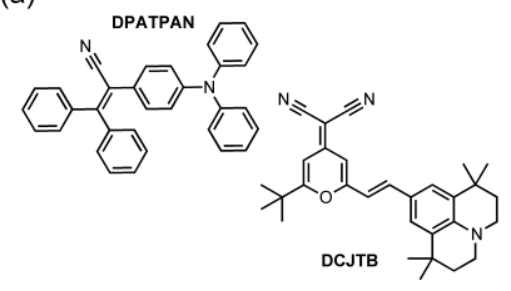

(c)

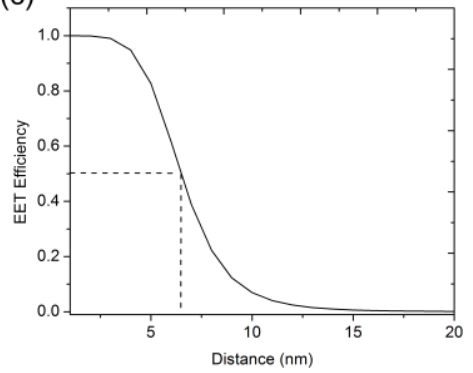

(b)

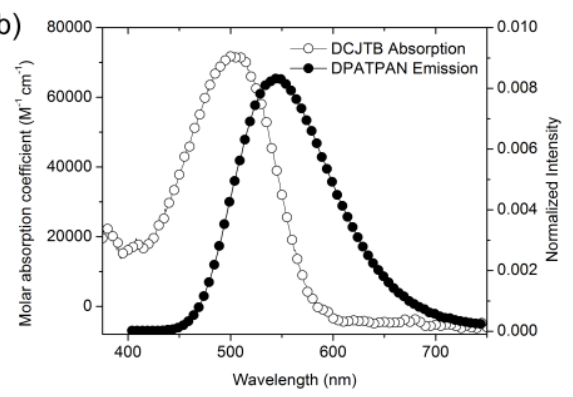

(d)

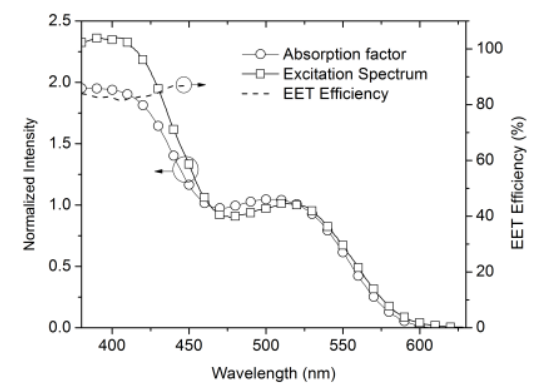

Figure 1. (a) Chemical structure of DPATPAN and DCJTB. (b) Absorption spectrum of DCJTB in molar (decadic) absorption coefficient units $\left(\mathrm{M}^{-1} \mathrm{~cm}^{-1}\right.$ ) and emission spectrum of DPATPAN with spectral area normalized to 1. (c) The EET efficiency $E$ as a function of interchromophore distance between donor and acceptor $\left(R_{D A}\right), E=R_{0} /\left(R_{0}+R_{D A}\right)$ estimated using the critical radius determined (see text). (d) EET efficiency of films estimated by taking the 5 ratio of the absorption factor $\left(1-10^{-\mathrm{A}(\lambda)}\right)$ spectrum and excitation spectrum, both normalized to 1 at the DCJTB absorption peak $(\sim 500 \mathrm{~nm})$

Recently, our group has demonstrated that materials exhibiting aggregation-induced emission (AIE) behaviour ${ }^{1-13}$ can be used to overcome the reabsorption problem in LSCs. ${ }^{10}$ The twisted aromatic hydrocarbon AIE materials investigated have unusually 10 large Stokes shifts. By using here an AIE donor chromophore with a more red-shifted emission and the EET approach, an LSC can be constructed with output closer to the band gap of highperformance solar cells.

The EET donor, DPATPAN, has properties typical of AIE 15 materials - the fluorescence quantum yield (c) of DPATPAN in the solid-state is $100 \%$ compared to $0.80 \%$ in tetrahydrofuran solution. ${ }^{14}$ In addition, DPATPAN exhibits a large Stokes shift with a small spectral overlap between absorption and emission (Figure S1). There are several key advantages for the 20 DPATPAN-DCJTB system in comparison to the previously reported rubrene-DCJTB. ${ }^{8}$ Unlike rubrene, DPATPAN can be used in high concentration such that the distance between DPATPAN and DCJTB are within the energy transfer critical distance while concomitantly avoiding concentration quenching. 25 In addition, it is noteworthy to mention that other EET systems not using AIE materials also have to compromise between FRET efficiency, quantum yield, reabsorption, and amount of light absorbed. ${ }^{6,9}$ The spectral matching of DPATPAN with DCJTB is better than rubrene. Rubrene has an absorption band that overlaps 30 with the DCJTB absorption causing both rubrene and DCJTB to reabsorb emission from DCJTB. ${ }^{15}$ In order to make comparisons with the rubrene LSC system published previously, ${ }^{8}$ attempts were made to fabricate rubrene/PMMA films $(30 \% \mathrm{w} / \mathrm{w}$ rubrene in PMMA) from solution. Interestingly, rubrene seems to be 35 unstable when solution processed in ambient conditions as the absorption spectrum of the dried rubrene/PMMA film did not show the typical absorption features of rubrene, which is possibly due to photo-oxidation of rubrene in air. ${ }^{16}$ DPATPAN, however, can be processed in air without any stability issues. This is the 40 first report of the use of AIE compounds as light-harvesting donors in an EET-based LSC.

Energy transfer characteristics of DPATPAN/DCJTB. With an emission maximum at $500 \mathrm{~nm}$, DPATPAN fulfils the energetic requirements for efficient energy transfer to the high fluorescence 45 quantum yield acceptor dye, DCJTB, making these compounds an excellent combination for the EET strategy. The energy transfer critical radius was estimated using the Förster equation: ${ }^{17}$

$$
R_{0}=0.02108\left(\frac{{ }^{2}{ }_{D} J}{n^{4}}\right)
$$

where $R_{0}(\mathrm{~nm})$ is the Förster critical distance, $\kappa^{2}$ is the orientation ${ }_{50}$ factor, $\Phi_{\mathrm{D}}$ is the quantum yield of the EET donor in the absence of the acceptor, $J\left(\mathrm{~nm}^{4} \mathrm{M}^{-1} \mathrm{~cm}^{-1}\right)$ is the spectral overlap between the absorption coefficient of the acceptor and normalized (area) emission spectrum of the donor, and $n$ is the refractive index of the medium. The absorption spectrum of DCJTB in units of 55 molar (decadic) absorption coefficient $\left(\mathrm{M}^{-1} \mathrm{~cm}^{-1}\right.$, with density of poly(methyl methacrylate) assumed as $1.17 \mathrm{~g} \mathrm{~cm}^{-3}$ ), and the area normalized emission spectrum of DPATPAN are shown in Figure 1b. The estimated $R_{0}$ for the DCJTB/DPATPAN EET pair is $5.3 \mathrm{~nm}$ using a refractive index for PMMA of $1.49, \kappa^{2}$ of ${ }_{60} 2 / 3$ (for random donor and acceptor transition dipole orientations), and measured quantum yield of DPATPAN of $92 \%$ (see Supporting Information). The Förster critical distance for our system is larger than a previously reported EET system (c.f. $R_{0}$ for [2-[2-[4-(dimethylamino)phenyl]ethenyl]-6-methyl-4H${ }_{65}$ pyran-4-ylidene]-propanedinitrile (DCM) / perylene-1,8,7,12tetraphenoxy-3,4,9,10-tetracarboxylic acid bis-(2',6'- 
diisopropylanilide) (LR305) is $4.0 \mathrm{~nm})^{6}$ mainly as a result of the higher quantum yield of DPATPAN (92\%) compared to (DCM) $(76 \%)$.

DCJTB/DPATPAN films on glass were then prepared by 5 dissolving the chromophores and the polymer matrix, poly(methyl methacrylate) (PMMA, weight average molecular weight $\sim 350 \mathrm{~kg} / \mathrm{mole}$ ), in chloroform followed by drop-casting on a glass substrate. The solvent was evaporated slowly in a covered Petri dish to increase the vapour pressure of the solvent 10 under ambient conditions (in air at $22{ }^{\circ} \mathrm{C}$ ). PMMA films with DCJTB only were similarly prepared. Due to the large Stokes shift and insensitivity to concentration quenching, it is possible to have a high concentration of the DPATPAN donor in PMMA for total light absorption while keeping the concentration of the 15 DCJTB acceptor in PMMA at a minimum to reduce the fluorescence reabsorption. More importantly, the concentration of DPATPAN can be very high without sacrificing energy transfer efficiency in contrast to a previously reported EET system. ${ }^{9}$ As a proof-of-principle, the weight ratio of donor and acceptor used to 20 investigate both EET and LSC efficiencies was set to 1:99 (w/w) to minimize the absorption contribution of DCJTB relative to DPATPAN. Efficient energy transfer was observed from DPATPAN to DCJTB when doped in PMMA. The estimated energy transfer efficiency based on the normalized absorption and 25 normalized excitation spectrum of the EET pair when doped in PMMA $(10 \% \mathrm{w} / \mathrm{w})$ is $86 \%$.

LSC fabrication optimization. The optimized film blend ratio, based on the measured quantum yield, was $10 \% \mathrm{w} / \mathrm{w}$ of the dye blend (DCJTB:DPATPAN 1:99) in PMMA $\left(\Phi_{\mathrm{F}}=92 \pm 1.5 \%\right)$. It 30 is important to note that the concentration of DCJTB relative to PMMA was kept at $0.1 \%(\mathrm{w} / \mathrm{w})$ to prevent fluorescence quenching of this acceptor dye due to aggregation. Film optimization details can be found in the Supporting Information. We have also attempted to fabricate LSCs using 35 DCJTB/DPATPAN without the PMMA polymer matrix. However, the resulting quantum yields of the pure DCJTB/DPATPAN-films were not as high as the $10 \%$ doping in PMMA (see Supporting Information for details).

Monte Carlo ray tracing analyses. The performance of the 40 DCJTB/DPATPAN LSC films was evaluated using Monte Carlo ray tracing simulations. Recently, our laboratory ${ }^{10,18}$ and other groups ${ }^{19,20}$ have shown that ray tracing simulations that include stochastic absorption, emission, and reabsorption events can provide insights on LSC performance, particularly the effects of 45 reabsorption. Two different conditions possible with our LSC were simulated: (1) when there is efficient light absorption at the donor dye DPATPAN absorption peak (absorbance of 1 at 400 $\mathrm{nm}$ ) and (2) when there is efficient light absorption at the acceptor dye DCJTB absorption peak (absorbance of 1 at 500 $50 \mathrm{~nm}$ ). In condition (1), both transmission and reabsorption were minimized since the donor absorption is high while the tail (acceptor) absorption is low. In contrast, condition (2) simulates an LSC that has a high contribution from the tail absorption due to DCJTB, which introduces significant reabsorption when light

55 propagates through the LSC. An LSC with absorbance of 1 at the absorption maximum is a practical value that can be readily achieved to reduce transmission losses for light harvesting. LSC devices, consisting of a chromophore/PMMA (nPMMA $=1.49$ ) layer on a $0.1 \mathrm{~cm}$ thick glass substrate ( $\mathrm{n}_{\text {glass }}=1.52$ ), were ${ }_{60}$ simulated to probe the influence of device area on efficiency. Edge reflections were also accounted for in all simulations. The UV-vis absorption and photoluminescence spectrum of the dye/PMMA films in Figure 2a were used as spectral inputs with $\Phi_{\text {F set }}$ at $92 \%$.

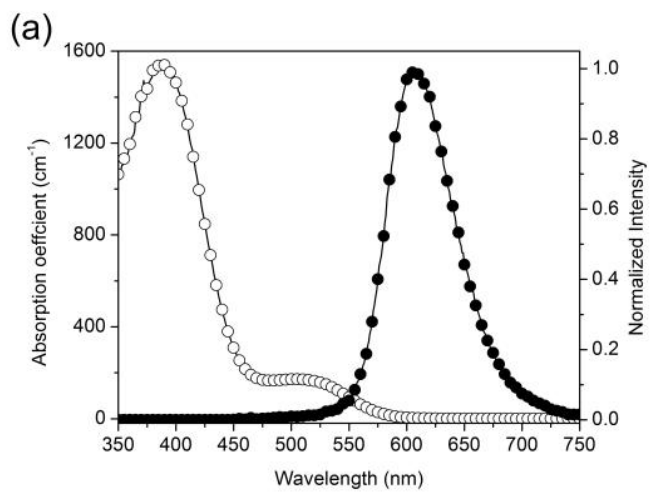

(b)

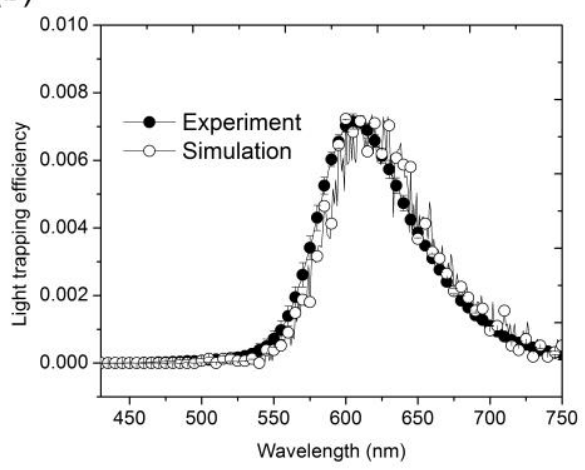

(c)

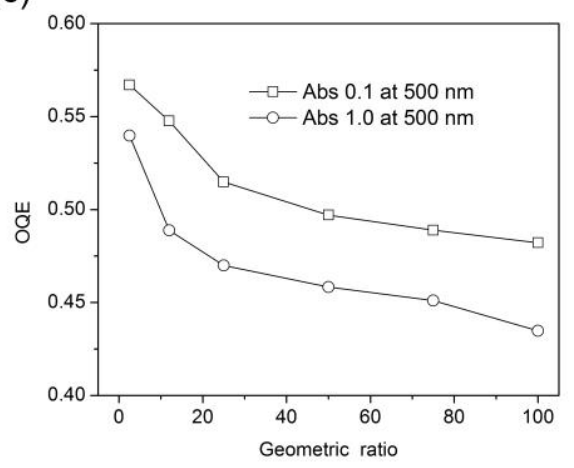

Figure 2. (a) Absorption spectrum in absorption coefficient units $\left(\mathrm{cm}^{-1}\right)$ and photoluminescence spectrum of (1:99 DCJTB:DPATPAN) 10\% in PMMA. (b) Experimental and simulated light trapping efficiency as a function of wavelength: $\left[I_{\text {total }}(\quad) I_{\text {face }}()\right] / \int I_{\text {total }}() d$, where

$70 I_{\text {total }}(\lambda)$ is the emission intensity of the whole LSC and $I_{\text {face }}(\lambda)$ is the emission intensity when the edges of the LSC are blocked with black matte paint. (c) Simulated OQE of the blend LSC at different acceptor absorbance. Only the thickness of the PMMA film was varied to achieve the different absorbance in the LSCs while keeping the concentration of 75 the chromophores in the film the same. A total of 100,000 photons for every geometric ratio were used for the simulation. Geometric ratio is the ratio of the concentrator face area and the concentrator edge area. 
(a)

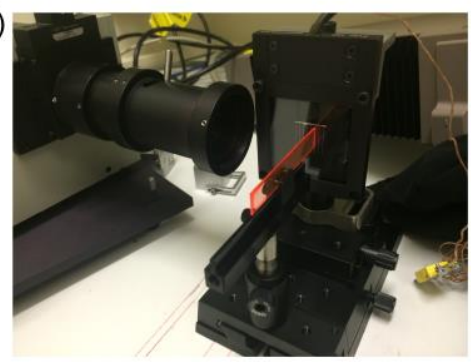

(d)

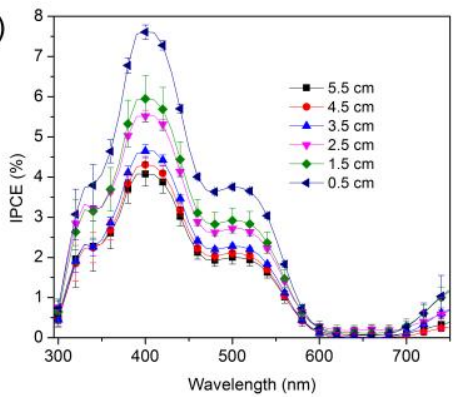

(b)

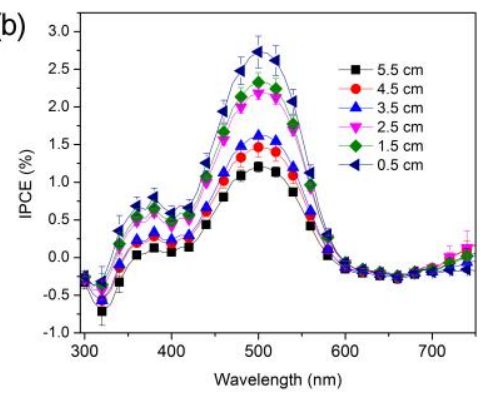

(e)

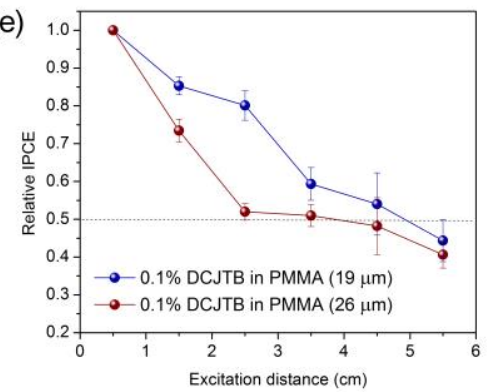

(c)
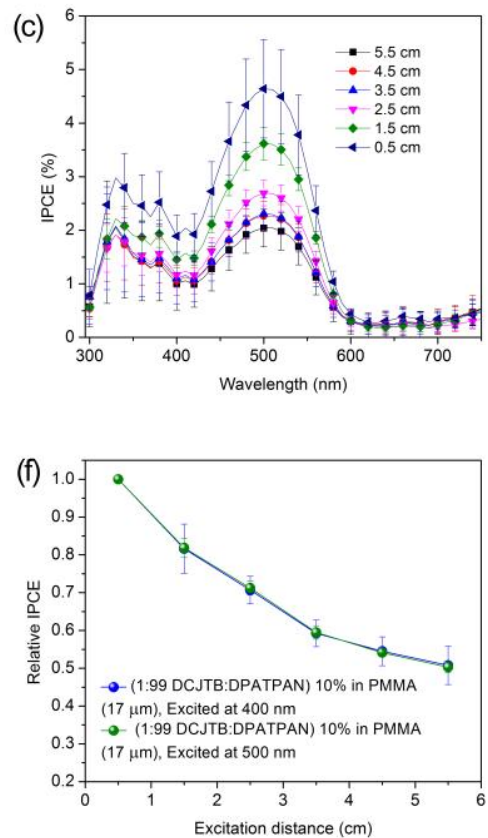

Figure 3. (a) Experimental set-up for distance-dependent IPCE measurements for the different LSCs investigated. Distance dependent IPCE of (b) $19 \mu \mathrm{m}$, (c) $26 \mu \mathrm{m}$ films of 0.1\% DCJTB in PMMA, (d) $17 \mu \mathrm{m}$ (1:99 DCJTB:DPATPAN) 10\% in PMMA. (e) Relative IPCE absorption maximum as a function of distance for DCJTB 0.1\% in PMMA films. (f) Relative IPCE absorption maximum as a function of distance for (1:99 DCJTB:DPATPAN) 10\% in PMMA 5 films excited at 400 and $500 \mathrm{~nm}$.

The trapping efficiency and optical quantum efficiency (OQE) of small LSCs of (DCJTB:DPATPAN 1:99) 10\% in PMMA, fabricated by drop casting on glass with dimensions $1.2 \mathrm{~cm} \times 1.2$ $\mathrm{cm} \times 0.1 \mathrm{~cm}$, were also measured according to the procedure 10 outlined by Mulder and co-workers. ${ }^{21}$ The edges emissions were differentiated from face emissions by blocking the edges with a black matte paint. Trapping efficiency is defined as the ratio of photons emitted from the edges to the total photons emitted from the LSCs and optical quantum efficiency (OQE) is the ratio of 15 photons emitted from the edges of the LSC to the total number of absorbed photons. The small LSCs gave experimental trapping efficiency of $63.3 \pm 2.2 \%$ ( $65 \%$ by simulation) and OQE of 58.2 $\pm 3.6 \%$ ( $58 \%$ by simulation). There is close agreement between measured and simulated in both LSC figures of merit, which ${ }_{20}$ provides evidence that simulations can provide results that have physical meaning (see also Figure 2b). The simulated OQE at different wavelengths as a function of geometric ratio shows that having a higher absorption contribution of the acceptor dye (absorbance of 1 at $500 \mathrm{~nm}$ ) leads to higher reabsorption losses. 25 In contrast, high donor dye absorption (absorbance of 1 at 400 $\mathrm{nm}$ ) with low acceptor dye absorption (absorbance of 0.12 at 500 $\mathrm{nm}$ ) allowed efficient light harvesting and decreased reabsorption loss (Figure 2c). This EET strategy meant that the LSC performance decay with increasing geometric ratio was 30 significantly less for high donor dye loading compared to systems with high acceptor dye loading. In contrast to other EET systems wherein both the donor and acceptor have low concentrations and low film absorbance to reduce the effects of concentration quenching and reabsorption, ${ }^{6}, 9$ there is no requirement for 35 compromise between film absorbance, chromophore concentration, and reabsorption in the system proposed here.
Distance-dependent incident photon conversion efficiency (IPCE). The performance of LSCs using the DCJTB/DPATPAN system was further evaluated by coupling the LSC with a 40 photovoltaic device. The incident photon conversion efficiency (IPCE) of the photovoltaic cell was monitored while varying the distance between a narrow excitation beam and the edge of the LSC (Figure 3a). An LSC with dimensions of $1.2 \mathrm{~cm} \times 7.5 \mathrm{~cm} \times$ $0.1 \mathrm{~cm}$ was used for the IPCE measurement. The films were 45 excited using a $325 \mathrm{~W}$ Hg light source (Newport IPCE Measurement Kit) with a spot size of $0.5 \mathrm{~cm}$ to simulate a point excitation source along a straight path. The IPCE instrument was calibrated with a standard silicon solar cell provided by Newport. The LSC and the same standard silicon solar cell were mounted 50 on a stage with a Vernier scale for distance measurement accuracy. The IPCE spectrum at each point on the film was measured by moving the stage by $1 \mathrm{~cm}$ intervals.

Apart from examining distance dependence, LSCs with different thicknesses of $0.1 \%(\mathrm{w} / \mathrm{w})$ DCJTB only in PMMA were also ${ }_{55}$ fabricated to provide a comparison with the DCJTB/DPATPAN system in PMMA. Given the power of the IPCE light source, a minimum film absorbance of $\sim 0.2$ at the maximum absorption peak was required to produce a measurable IPCE signal at the farthest excitation distance of $5.5 \mathrm{~cm}$ from the solar cell. The ${ }_{60}$ IPCE spectra as a function of distance of the DCJTB/PMMA films with 19 and $26 \mu \mathrm{m}$ film thickness are shown in Figure 3b and Figure 3c, respectively (see Figure $\mathbf{S 4}$ for absorbance spectra of different thicknesses of the DCJTB/PMMA films). The IPCE spectrum of the films have a similar spectral profile to the 65 absorbance spectrum of DCJTB, which confirms that the photocurrent response originates from the LSC and not from trapped scattered light from the lamp. The relative IPCE 
performance decreases as a function of distance for both films although the IPCE decay was more significant for the thicker 26 $\mu \mathrm{m}$ film with higher absorbance (Figure 3e). The critical length, defined as the distance at which the IPCE drops by $50 \%$, was 5 5 and $4 \mathrm{~cm}$ for the 19 and $26 \mu \mathrm{m}$ films respectively at $500 \mathrm{~nm}$ excitation. While the $19 \mu \mathrm{m}$ films showed less reabsorption loss, the transmission loss was higher resulting in a lower photocurrent detected and, hence, lower peak IPCE. The IPCE at $500 \mathrm{~nm}$ of the thicker $26 \mu \mathrm{m}$ film at $0.5 \mathrm{~cm}$ was almost double the IPCE of 10 the $19 \mu \mathrm{m}$ film (Figure 3b and Figure 3c).

The IPCE of the DCJTB/DPATPAN in PMMA film is shown in Figure 3d. DCJTB/DPATPAN films (17 $\mu \mathrm{m}$ thickness) were fabricated with similar absorbance at $500 \mathrm{~nm}$ to that of the $19 \mu \mathrm{m}$ DCJTB/PMMA film. The IPCE decay as a function of excitation 15 distance for the DCJTB/DPATPAN films was comparable to the $19 \mu \mathrm{m}$ DCJTB/PMMA film since reabsorption only arises from the acceptor. On the other hand, the high absorbance of the DPATPAN donor dye greatly reduced transmission loss leading to a substantially improved light harvesting performance. This 20 improvement was clearly observed in the IPCE spectrum, particularly at $400 \mathrm{~nm}$, the maximum absorption wavelength of DPATPAN (Figure 3d). The IPCE decay of the DCJTB/DPATPAN films was also compared using 400 and 500 $\mathrm{nm}$ excitation (Figure 3f). Similar behaviour observed at the two 25 wavelengths is consistent with energy transfer between the DPATPAN and DCJTB dyes.

Short-circuit current dependence on distance. The shortcircuit current $\left(J_{\mathrm{sc}}\right)$ of a solar cell can be calculated from the IPCE data by integrating the product of the AM1.5G photon flux and 30 the IPCE spectrum. The integrated IPCE provides a better comparison of the improved light harvesting of the DCJTB/DPATPAN blend films compared with the DCJTB/PMMA LSCs. The $J_{\text {sc }}$ of the DCJTB/DPATPAN LSC is substantially higher compared to both the 19 and $26 \mu \mathrm{m}$ thick

${ }_{35}$ DCJTB/PMMA films (Figure 4a) as a significant amount of light was harvested by the DPATPAN dye. The decay of $J_{\mathrm{sc}}$ as a function of excitation distance in Figure $4 \mathbf{b}$ shows a similar trend as for the IPCE result. The critical length for $J_{s c}$ for the $26 \mu \mathrm{m}$ thick DCJTB/PMMA film of $2.5 \mathrm{~cm}$ is significantly shorter than 40 that of the DCJTB/DPATPAN LSC at $4 \mathrm{~cm}$.

\section{Conclusions}

In summary, we have demonstrated an EET strategy involving donor and acceptor chromophores that have well separated absorption and exhibit efficient energy transfer properties in ${ }_{45}$ PMMA substrates. The efficient EET process observed in the PMMA films allows for a large donor to acceptor chromophore ratio in LSC devices. Using this approach the absorption contribution of the acceptor can be kept relatively low leading to small reabsorption losses while maintaining effective light 50 harvesting by the donor, which is unprecedented for fully solution-processed all-organic LSCs. The AIE-type chromophore was not susceptible to fluorescence quenching at high concentrations, which also contributes to the efficient energy transfer observed in the PMMA films. Typical laser dyes that 55 exhibit fluorescence quenching at high concentrations would have reduced energy transfer performance due to the dependence of EET on the donor fluorescence lifetime. The EET strategy reported here represents a significant step towards LSCs that harvest light efficiently.
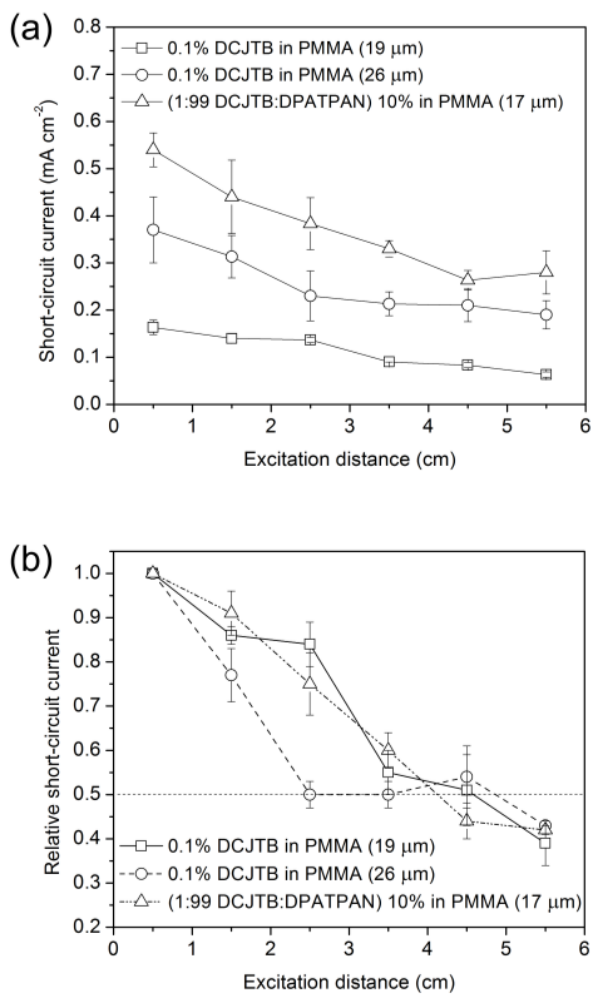

${ }^{60}$ Figure 4. (a) Estimated short-circuit current when the Si solar cell is illuminated by the different LSCs. (b) Relative short-circuit current as a function of distance for the different LSCs.

\section{Acknowledgements}

${ }_{65}$ This work was made possible by support from the Australian Renewable Energy Agency which funds the project grants within the Australian Centre for Advanced Photovoltaics. WWHW is supported by an Australian Research Council Future Fellowship (FT130100500) and JLB is supported by an Australian 70 Postgraduate Award. Responsibility for the views, information or advice expressed herein is not accepted by the Australian Government.

\section{Notes and references}

Bio21 Institute, School of Chemistry, The University of Melbourne, 75 Parkville 3010 Australia; E-mail: ghiggino@unimelb.edu.au (for KPG), wwhwong@unimelb.edu.au (for WWHW)

$\dagger$ Electronic Supplementary Information (ESI) available: Experimental details, absorption spectra of individual chromophores in PMMA, energy transfer characterization, blend optimization of pure /PMMA dispersed

80 films, and absorbance spectra of LSC can be found in the Supporting Information.

See DOI: $10.1039 / \mathrm{b} 000000 \mathrm{x} /$

1. W. H. Weber and J. Lambe, Appl. Opt., 1976, 15, 2299-2300.

85 2. L. R. Wilson, B. C. Rowan, N. Robertson, O. Moudam, A. C. Jones and B. S. Richards, Appl. Opt., 2010, 49, 1651-1661.

3. R. Ziessel, G. Ulrich, A. Haefele and A. Harriman, J. Am. Chem. Soc., 2013, 135, 11330-11344. 
4. J. Iehl, J. F. Nierengarten, A. Harriman, T. Bura and R. Ziessel, J. Am. Chem. Soc., 2012, 134, 988-998.

5. O. A. Bozdemir, S. Erbas-Cakmak, O. O. Ekiz, A. Dana and E. U. Akkaya, Angew. Chem. Int. Ed., 2011, 50, 10907-10912.

5 6. B. Balaban, S. Doshay, M. Osborn, Y. Rodriguez and S. A. Carter, $J$. Lumin., 2014, 146, 256-262.

7. S. T. Bailey, G. E. Lokey, M. S. Hanes, J. D. M. Shearer, J. B. McLafferty, G. T. Beaumont, T. T. Baseler, J. M. Layhue, D. R. Broussard, Y.-Z. Zhang and B. P. Wittmershaus, Sol. Energy Mater. Sol. Cells, 2007, 91, 67-75.

8. M. J. Currie, J. K. Mapel, T. D. Heidel, S. Goffri and M. A. Baldo, Science, 2008, 321, 226-228.

9. Y. Shen, Y. Jia, X. Sheng, L. Shen, J. A. Rogers and N. C. Giebink, ACS Photonics, 2014.

15 10. J. L. Banal, J. M. White, K. P. Ghiggino and W. W. H. Wong, Sci Rep, 2014, 4, 4635.

11. J. Mei, Y. Hong, J. W. Y. Lam, A. Qin, Y. Tang and B. Z. Tang, Adv. Mater., 2014, 26, 5429-5479.

12. Y. Hong, J. W. Y. Lam and B. Z. Tang, Chem. Soc. Rev., 2011, 40, 5361-5388.

13. Y. Hong, J. W. Y. Lam and B. Z. Tang, Chem. Commun., 2009, 4332-4353.

14. Y. Gong, Y. Tan, J. Liu, P. Lu, C. Feng, W. Z. Yuan, Y. Lu, J. Z. Sun, G. He and Y. Zhang, Chem. Commun., 2013, 49, 4009-4011.

25 15. H. Mattoussi, H. Murata, C. D. Merritt, Y. Iizumi, J. Kido and Z. H. Kafafi, J. Appl. Phys., 1999, 86, 2642-2650.

16. M. Kytka, A. Gerlach, F. Schreiber and J. Kovac, Appl. Phys. Lett., 2007, 90.

17. B. W. v. d. Meer, in FRET - Förster Resonance Energy Transfer:

30 From Theory to Applications, eds. I. Medintz and N. Hildebrandt, Wiley-VCH Verlag GmbH \& Co. KGaA, Weinheim, Germany, 2014, pp. 23-59.

18. C. Haines, M. Chen and K. P. Ghiggino, Sol. Energy Mater. Sol. Cells, 2012, 105, 287-292.

35 19. F. Meinardi, A. Colombo, K. A. Velizhanin, R. Simonutti, M. Lorenzon, L. Beverina, R. Viswanatha, V. I. Klimov and S. Brovelli, Nature Photon., 2014, 8, 392-399.

20. N. D. Bronstein, L. Li, L. Xu, Y. Yao, V. E. Ferry, A. P. Alivisatos and R. G. Nuzzo, ACS Nano, 2013, 8, 44-53.

40 21. C. L. Mulder, P. D. Reusswig, A. M. Velázquez, H. Kim, C. Rotschild and M. A. Baldo, Opt. Express, 2010, 18, A79-A90. 